\title{
Application of a Memristive Neural Network for Classification of COVID-19 patients
}

\author{
Stoyan Kirilov ${ }^{1}$, Violeta Todorova ${ }^{1}$, Ognyan Nakov ${ }^{2}$, Valeri Mladenov ${ }^{1}$ \\ ${ }^{1}$ Department of Theoretical Electrical Engineering, ${ }^{2}$ Department of Computer Systems \\ Technical University of Sofia \\ 1000 Sofia, 8 Kl. Ohridski Blvd \\ Bulgaria \\ s_kirilov@tu-sofia.bg
}

Received: January 28, 2021. Revised: August 4, 2021. Accepted: August 29, 2021. Published: August 31, 2021.

\begin{abstract}
The global pandemic of COVID-19 has affected the lives of millions around the globe. We learn new facts about this corona virus every day. A contribution to this knowledge is described in the paper and it is related to employment of memristor neural networks and algorithms that help us analyze patients' data and determine what patients are at increased risk for developing severe medical conditions once infected with the COVID-19. An efficient separation of potential patients in ill and healthy sub-groups is conducted using software and hardware neural networks, machine learning and unsupervised clustering. In the recent years, many works are related to reducing of neural chips area for the hardware realization of neural networks. For this purpose, a partial replacement of CMOS transistors in neural networks by memristors is made. Some of the main memristor advantages are its lower power consumption, nano-scale sizes, sound memory effect and a good compatibility to CMOS technology. In this reason, the main purpose of this paper is application of a memristor-based neural network with tantalum oxide memristor synapses for COVID-19 analysis. Additional experiments with data clustering are conducted. Experiments show that in fact patients with specific underlying health conditions and indicators are more predisposed to develop severe COVID-19 illness. This research is helpful for engineers and scientists to easier identifying patients that would need medical help.
\end{abstract}

Key-Words: COVID-19, pandemic, tantalum oxide memristor, synapses, neural network, machine learning, unsupervised learning, SARSCoV-2.

\section{INTRODUCTION}

Without doubt, COVID-19 has been the most covered topic for over a year now in every country in the world. The corona virus has reached almost every corner of the globe, caused the death of almost three million people, and infected over one hundred million people around the world [1]. For almost a year, there has been no solution to the COVID-19 crisis, except national lockdowns and recommendations to practice social distancing. At the end of 2020, the scientists brought to the market the first vaccine against COVID-19. Several other vaccines have been also approved. The hope for everyone is that the vaccines will help alleviate the world from the pandemic. We learn new facts about COVID-19 every day and the more knowledge we acquire, the stronger we can get in the fight against it [1], [2]. We have conducted an experiment to categorize and determine what patients are at increased risk for COVID-19 virus. This research is focused on identifying the population of patients with specific underlying health conditions that are more prone to develop severe illness caused by COVID-19. The experiment has used memristorbased neural networks, to analyze patients' data and to classify the level of severity of the disease [2].

Corona viruses have been known to people as a family of viruses that can cause illnesses such as the common cold, severe acute respiratory syndrome (SARS) and Middle East respiratory syndrome (MERS) [1], [2]. Towards the end of 2019, a new coronavirus was identified as the cause of a disease outbreak that was first reported in humans in Wuhan, China [1], [3]. The virus is now known as the severe acute respiratory syndrome coronavirus 2 (SARS-CoV-2) and the disease it causes is called coronavirus disease 2019 (COVID-19) [1], [4]. The COVID-19 outbreak was declared as a pandemic by 
the World Health Organization (WHO) in March 2020 [1], [5]. The severity of COVID-19 symptoms can range from very mild to severe. Some patients may have only a few symptoms, while others may have no symptoms at all. Some people may experience more severe symptoms, such as shortness of breath and pneumonia. It has been observed that the risk of COVID-19 illness increases with age. People with pre-existing medical conditions, as cancer, diabetes, heart diseases and others may have a higher risk of developing a serious illness [1], [5], [6]. There have been several studies exploring the effects of SARS-CoV-2 on patients with specific pre-existing conditions [4], [5], [6]. Some researchers have employed different machine learning approaches to assess the risk factors on patients [4], [5], [7]. A study for identifying the characteristics predisposing COVID19 patients to in-hospital death was conducted in Italy. The used machine learning analysis revealed that impaired renal function, elevated C-reactive protein, and advanced age were major predictors of in-hospital death in a large cohort of patients with COVID-19 [1], [4], [5]. Another research concluded that individuals with chronic conditions such as diabetes, cardiovascular diseases (CVDs), and chronic obstructive pulmonary disease (COPD) are at increased risk of morbidity and mortality [4], [5], [7]. The information for pre-existing conditions gives the patients and their medical providers the opportunity to seek preventive solutions, as social distancing and national lockdowns till the pandemic is handled on a world scale [2], [5], [8].

Efficient techniques for evaluation of the main risk factors associated to development of COVID-19 disease and for its prediction are needed. The neural networks are very appropriate for separation of potentially healthy and ill patients and for approximate prediction the illness distribution in time domain [4], [5], [6], [8]. The neurons and synapses of the traditional networks are based on complementary metal-oxide-semiconductor (MOS) technology [9], [10], [11], [12]. In the recent years, neural networks based on both CMOS and memristors are analyzed to partially replace the MOS transistors with memristors, and to analyze their potential applications [9], [12], [13], [14].

The memristor element was postulated by Chua in 1971 [15] as the fourth fundamental passive electronic two-terminal element. It is realized and validated experimentally in Hewlett-Packard research labs by Williams in 2008 [16]. Memristors have a specific and well-expressed memory effect. Their resistance, namely memristance, is altered by applied external voltage pulses with certain level, polarity, and duration [12], [14], [17]. Moreover, the memristance is retained when the power supply is off [14]. This shows the memristor's non-volatility [14], [16]. The memristors have many advantages compared to the present MOS electronic elements, as nano-scale size, memory effect, low power consumption, which make them preferrable for building artificial hardware neural networks [18], [19], [20]. Since the neural networks are preferred for pattern recognition, the engineers and scientists are interested in optimizing their hardware [10], [16], [21]. In the last several years the present CMOS technology is approaching a bottleneck in terms of size. In this sense, the memristor elements based on perspective materials, as transition metal oxides and especially $\mathrm{Ta}_{2} \mathrm{O}_{5}$ are promising candidates to replace some of the MOS transistors in artificial neural networks [17], [22], [23]. This paper focuses on the application of a feed forward neural network with memristor-resistor synapses with a differential amplifier in identifying and classifying patients with specific underlying health conditions that are more predisposed to develop severe illness by SARS-CoV-2 and comparison the results to those obtained by machine learning and unsupervised clustering [4], [5], [6], [8].

The paper is organized as follows. In the next section the data collection and their description are discussed. In the next third section two approaches are presented - a supervised learning with neural networks (NN) and non-supervised - k-means clustering for classifying patients based on their underlying conditions. A neural network with memristor synapses, and a machine learning algorithm are considered and compared for the analyses, and a discussion of the results are presented in section 4 . The conclusion remarks are given in the final section five.

\section{Data Collection, Data Description AND DATA ClEANING}

Randomly selected patients' medical data used for training the applied feed-forward neural network have been employed. Two types of algorithms, namely supervised and unsupervised training, were applied in the work [9], [24]. For a supervised training, a set of data is used to train the neural network for recognizing subjects that are predisposed to COVID-19, tracking their development, and generation of a predictive model that can result in determining what patient with what specific pre-existing health condition may be at risk of developing severe illness due to SARS-CoV-2 infection. Target data containing the confirmed ill 
and healthy patients are used for the supervised learning technique. For unsupervised learning, a self-organizing map based on competition is applied, and the neural network groups the data so that the patients at risk and the healthy patients fall into different clusters [6], [8], [9], [24]. The considered self-organizing map is used for clustering the analyzed objects, based on their different statistical properties [9], [24]. The data used in this research has been provided by the Memorial Medical Center. The used file contains anonymized clinical information about patients, compliant with the General Data Protection Regulation (G.D.P.R.), along with longitudinal data from their electronic medical records (EMRs) [6].

The raw medical data consists of all the available information up to that moment, coming from clinical records, covering a period slightly longer than 10 months, from April 2020 until February 2021. Clinical records of 20400 potential patients were used, out of which 10200 patients have been infected with SARS-CoV-2, and the other 10200 hospital patients that have not been infected. The medical records are related to 10232 female and 10168 male patients, in age range from 21 to 85 years old [6], [7].

The row dataset contains seventeen medical parameters: anonymized patient ID, gender, age, COVID-19 status and severity, body mass index, heart disease status, hypertension status, diastolic and systolic blood pressure, diabetes status, hemoglobin values, chronic kidney disease status, calcium, potassium and phosphorus values, and cancer status [5], [6]. The extracted data are often incomplete or contain unnecessary information and noise that affect the performance of the applied predictive models. Thus, it is necessary to preprocess and validate them to avoid issues.

In this work, a statistical analysis software for deriving the needed variables into a suitable format is applied. A cross-validation of the data is conducted before training and testing the network for obtaining more accurate results. The presence of COVID-19 disease has been flagged by ' +1 ' and its absence is denoted by ' -1 '. The male gender is coded as ' 1 ' and the female one - by ' 0 '. The data has been transposed to one record per patient containing the described above medical parameters and indicators.

\section{NEURAL NETWORK FOR PATTERN RECOGNITION}

In this paper, we are interested in the artificial neural networks' learning and training [6], [8], [9], [10] for conduction of automatic analysis of electronic health records (EHRs), for categorizing patients with pre-existing health conditions and their predisposition to COVID-19 illness. Using the collected patients' dataset, two main techniques are applied - a supervised learning and k-means clustering to study the data for patients' classification for increased risk of SARS-CoV-2 illness based on their pre-existing health conditions and indicators [2], [3], [7]. These different techniques are applied for comparison and for deriving more realistic results. Memristor-based synaptic circuits with a current divider and complementary MOS transistors are applied in the considered feed forward artificial neural network for data classification.

\section{A. Supervised learning}

A feed-forward artificial neural network is applied for approximate evaluation of the main risk factors associated to people with COVID-19 illness and for approximate prediction of presence or absence of this disease in a selected group of potential patients. A set of 20400 potential patients is analyzed using the memristor neural network. For this test, 15 important medical features and indicators are applied (patient's gender, age, COVID-19 severity, body mass index, heart disease and hypertension status, diastolic and systolic blood pressure, diabetes and hemoglobin values, chronic kidney disease, calcium, potassium and phosphorus values, and cancer status).

These features are grouped in a matrix, which rows correspond to the described above medical properties and health conditions. Each column of the considered matrix is related to a given potential patient. The described matrix represents the input data for the artificial feed forward neural network. The first 14280 records (about $70 \%$ of the considered group of potential patients) are used for training the neural network. The other 6120 records of the matrix are applied for testing the memristor neural network after the training stage.

The target data are placed in a row of the used matrix. It contains "-1" for absence of COVID-19 and "+1" for a positive COVID-19 test, respectively. The target row contains 14280 elements for training and learning of the neural network. The other 6120 elements of the target row are used for testing the neural network and for validation the results. The memristor neural network 
contains an input layer of 15 nodes for applying the input signals, and two layers of neurons - a hidden layer and an output layer, respectively [9].

The fifteen input signals applied to the neural network correspond to the previously discussed medical features and indicators for classification of potential patients with COVID-19 disease.

The included 12 neurons in the hidden layer of the neural network have tangent sigmoidal activation function $h(x)$ [9], [10]:

$$
h(x)=\frac{\exp (x)-\exp (-x)}{\exp (x)+\exp (-x)}
$$

where $x$ is the weighted sum of the input signals $x_{i}$ :

$$
x_{j}=w_{j b}+\sum_{i=1}^{15} w_{i j} x_{i}
$$

and $w_{j b}$ is the weight of the corresponding synapse used for applying the unity bias signal [10]. The index $i$ represents the number of the respective input node and $j$ shows the number of the considered neuron in the hidden layer of the neural network. Each input signal is applied to all the neurons in the hidden layer using memristor-based synaptic devices. The outputs of the neurons in the hidden layer are connected to the neuron in the output layer. This neuron has a linear activation function. The actual output signal of the considered memristorbased neural network is denoted by $y[10]$ :

$$
y=v_{b}+\sum_{j=1}^{12} v_{j} x_{j}
$$

and the weight of the synaptic bond used for forwarding the respective bias signal is denoted by $v_{b}$. The error signal $e$ is equal to the difference between the actual output signal of the neural network $y$ and the desired output signal $d$, which is correspondent to the target row.

The obtained error signal is used for correction the weights of the memristor synapses between the neurons of the hidden layer and the output during the training process, in a combination with the previously determined learning rate $\eta$ and the signals zj, applied to the input of the neuron in the output layer of the artificial neural network [10]:

$$
\Delta v_{j}=\eta \cdot \delta \cdot z_{j}
$$

where $\delta$ is an error information term:

$$
\delta=(d-y) f^{I}(y)
$$

The weight update of the synapses between the input nodes and the neurons in the hidden layer is:

$$
\Delta w_{i j}=\eta \cdot \delta_{j} \cdot x_{i}=\eta \cdot x_{i} \cdot \sum_{j=1}^{12} \delta \cdot v_{j}
$$

A voltage signal proportional to the respective error signal is applied to the synapses for correction of their weights. The weight update is repeating till obtaining the minimal value of the mean square error between the desired output signal and the actual one. After minimizing the mean square error follows the usage stage of the memristor neural network. The derived error after the usage period is equal to the number of incorrectly classified patients divided by their total number and in the present case, it is about $1.96 \%$.

\section{B. $\quad k$-means clustering}

An additional analysis of the selected group of potential patients is conducted applying data clustering [10], [24]. It is helpful technique in exploratory data analysis and data mining in many fields of research. Data clustering has a good efficiency and low memory usage. In the nonsupervised learning of the neural network, a hidden statistical structure in data which we do not previously know is used. The analyzed data must be partitioned in clusters which number has to be previously defined. This information is often unavailable previously, so multiple trials must be conducted to find the optimal number of clusters. Data clustering is a method for unsupervised classification [9], [10]. The clusters are generated by evaluation of the similarities of several underlying characteristics of the analyzed data between several different cases. The grouping of the objects is based on the similarities between them. A reduction of the data complexity is applied by preliminary statistical analysis and data processing. In the clustering analysis, a natural grouping in data is derived, such that items in the same cluster are more similar one to another than those from the other clusters. The kmeans clustering technique is similar to an approximation of the commonly used mixture model with component extraction with maximal probability. In k-means clustering, each cluster is represented by a "prototype" data point. The kmeans clustering procedure can be described as a centroid model, where one multidimensional vector represents the mean value of a characteristic of the grouped objects, and it is also used for representation of each data cluster. Different metrics are applicable for evaluation the similarity between the clusters, as the Euclidian distance, the squared Euclidian distance, the sum of absolute differences, cosine and Hamming metrics, and others [10], [24]. The mainly used in data clustering squared Euclidian distance is expressed as follows [10]: 


$$
d E^{2}=\sum_{k=1}^{n}\left(c_{k}-x_{k}\right)^{2}
$$

where $c$ represents the center of the considered cluster, $x$ is related to the coordinates of the analyzed data point, $k$ is the dimension of the related data point in the considered multidimensional space, and $n$ is its maximal dimension [10]. The considered multidimensional space is partitioned in several subspaces, which represent the data clusters. This clustering procedure minimizes the sum of the point-to-cluster-centroid distances, over all subspaces. The algorithm of data clustering is based on initialization and calculation of the coordinates of the centroids of the subspaces [10].

When the centroid of a data cluster belongs to the nearest one, no changes are made. If another centroid is the nearest one, according to the calculated coordinates, then the considered cluster is reassigned to the other centroid and the current coordinates for the old and the new subspaces are recalculated as the mean of the described data clusters [10], [24]. The main advantage in the present case is that we previously know that the number of clusters is two (patients with COVID-19 symptoms and without them, respectively).

The data clustering is realized in MATLAB environment [25]. The obtained error after the classification is about $2.3 \%$. Additional experiments for data clustering are also performed in two, three and four classes, respectively [10], [25]. The best data clustering is obtained if the analyzed data are grouped in two classes. The learning time of the neural network in this case is about $2983 \mathrm{~ms}$. After the conducted analyses it is obtained that COVID-19 illness is predominantly observable in patients older than 65 years and with systolic blood pressure from 125 to 130 .

\section{The considered memristor-based neural network}

Many scientific works are related to synaptic device realizations [10], [19], [20], [21], [26]. The traditional synapses of the neural networks are based on electronic elements generated in CMOS technology. Several suitable and perspective memristor-based synaptic circuits are described as well [19], [20], [21], [26].

Some of these devices are able to realize only positive synaptic weights. The memristor-based bridge synaptic circuits can produce positive, zero and negative weights [12], [14]. Another memristorbased synaptic circuit with an operational amplifier and two memristors per synapse is described in [13], [19]. It is programmed by applied external short voltage pulses.
In this work, a different synapse based on a current divider with a memristor, resistors and differential amplifier with MOS transistors, is applied. This synaptic scheme is with a reduced number of elements. It contains only one memristor element per synapse. It realizes positive, zero and negative synaptic weights.

The tantalum oxide memristor $M$ is applied in the synaptic circuit, owing to its good memory effect and sound switching properties. The memristor element has two terminals - top electrode (TE) and bottom electrode (BE), respectively [17], [23]. Its nanostructure has a square cross-section. It contains several parallel conducting channels [17], [23]. The peripheral region of the memristor is made of pure and stochiometric $\mathrm{Ta}_{2} \mathrm{O}_{5}$ amorphous material. The central conducting channel of the memristor is based on a solid solution of oxygen in tantalum material. Another channel of doped with oxygen vacancies tantalum oxide is formed between these two regions [17].

The memristor state is changed by applied external packages of positive or negative rectangular voltage pulses with a duration of $1 \mu$ s and a level of $0.52 \mathrm{~V}$. A schematic of the considered memristorbased neural network is shown in Fig. 1.

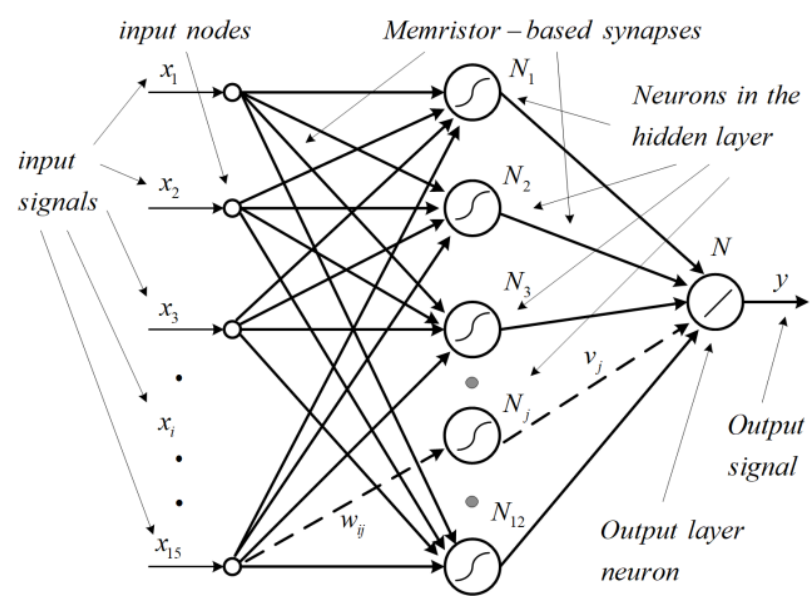

Fig. 1 A schematic of the considered memristorbased feed forward neural network

The applied modified memristor model [20] has improved characteristics according to the standard Hewlett-Packard model [17]. The used tantalum oxide memristor model is considered and described in details in [26], [27]. Its corresponding LTSPICE library model applied in the work is freely available at: https:/github.com/ mladenovvaleri/AdvancedMemristor-Modeling-in-LTSpise [26].

The resistances of the memristor elements, included in the synapses between the neurons in the hidden layer of the network and the output neuron 
are presented in Table 1. The number of the respective neuron in the hidden layer is denoted by the index $j$, which changes from 1 to 12 . The values of the corresponding memristances are obtained after the training stage of the artificial neural network. The memristance $M$ in this case changes between $300 \Omega$ and $783 \Omega$. The values of the memristance, which are higher than $300 \Omega$ correspond to positive synaptic weights.

Table 1. Memristances corresponding to the weights after the training process for the synapses $v_{i j}-$ between the hidden layer and the output

\begin{tabular}{|l|c|c|c|c|c|c|}
\hline \multicolumn{7}{|c|}{ Memristances related to the synapses Vij } \\
\hline Neuron & 1 & 2 & 3 & 4 & 5 & 6 \\
\hline$M$ & 435,34 & 300,00 & 300,00 & 300,00 & 300,00 & 782,93 \\
\hline Neuron & 7 & 8 & 9 & 10 & 11 & 12 \\
\hline M & 300,00 & 300,00 & 300,00 & 300,00 & 300,00 & 300,00 \\
\hline
\end{tabular}

The memristances related to the synapses placed between the input nodes and the hidden layer are presented in Table 2. The respective input nodes are denoted from 1 to 15 and they correspond to the medical indicators discussed above. The memristances are derived after the training of the neural network. In the present case the memristance is changing between $92 \Omega$ and $1930 \Omega$. Memristances lower than $300 \Omega$ correspond to negative synaptic weights [26], [28].

Table 2. Memristances corresponding to the synaptic weights after the training for the synapses $w_{i j}$ - between the input layer and the hidden layer

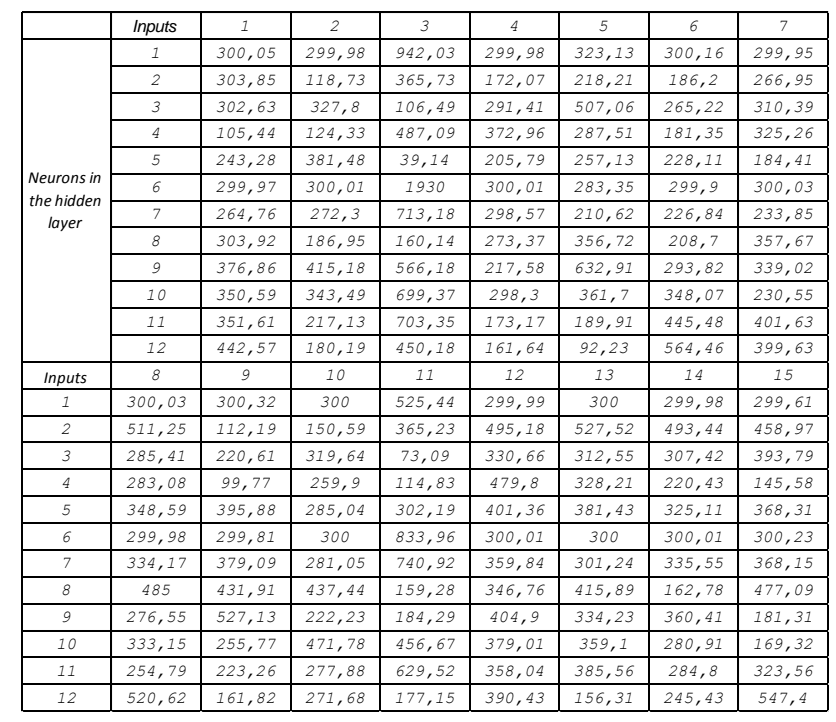

A schematic of the considered modified synaptic circuit is given in Fig. 2. The applied synapse contains a current divider (CD) and a differential amplifier realized with MOS transistors $-T_{1}$ and $T_{2}$. The used current divider contains two branches, connected in parallel. The first branch of the current divider contains a memristor $M$ and a resistor $-R_{4}$, and the second one has two resistors, connected in a series $-R_{l}$ and $R_{5}$. The corresponding currents flowing through the considered branches are denoted by $i_{1}$ and $i_{2}$. The input voltage signal of the synaptic circuit is $v_{i n}$. The respective transfer coefficient of the differential amplifier is denoted by $k_{v}$ and it has a value of 10 . The resistance of the element $R_{l}$ has a value of $300 \Omega$, and the resistors $R_{4}$ and $R_{5}$ included in the current divider are with resistances of $1 \mathrm{k} \Omega$.

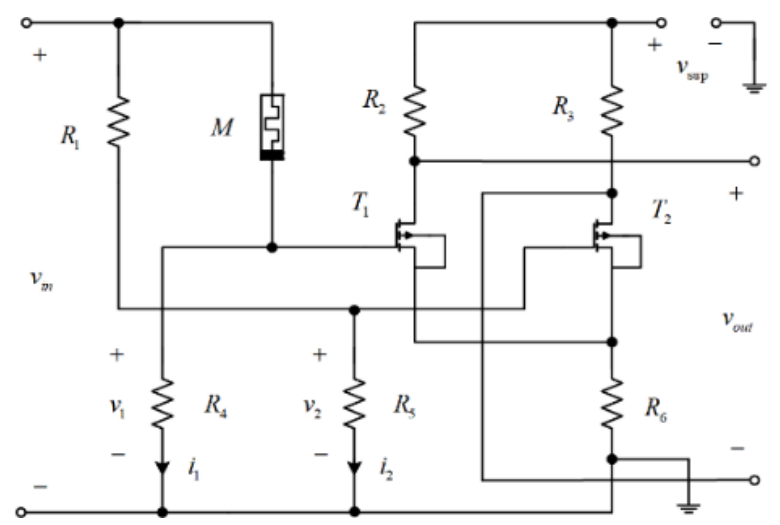

Fig. 2 A schematic of the applied synapse based on tantalum oxide memristor

The operation of the described memristor-based synaptic scheme is based on comparison of the currents $i_{1}$ and $i_{2}$ flowing through the branches of the current divider [26]. By altering of the memristance $M$, the synaptic weight $w$ is changed during the weight update stage. After analysis of the memristor-based synaptic scheme, the weight $w$ dependent on the memristance $M$ is derived:

$$
w(M)=\frac{v_{\text {out }}}{v_{\text {in }}}=k_{v} \cdot\left(\frac{R_{5}}{R_{1}+R_{5}}-\frac{R_{4}}{M+R_{4}}\right)
$$

The memristance $M$ and the corresponding synaptic weight of the circuit $w$ are changed by applying of external voltage pulses. After transformation of equation (1) and having in mind that the resistance $R_{4}$ is equal to $R_{5}$, the next expression is obtained:

$$
\mid \begin{aligned}
& w(M)=0, M=R_{1} \\
& w(M)<0, M<R_{1} \\
& w(M)>0, M>R_{1}
\end{aligned}
$$

The resistance of the used tantalum oxide memristor $M$ as a function of the applied voltage $v$ and the state variable $x$ could be expressed by the applied modified memristor model as follows [27]: 


$$
M(x, v)=\frac{1}{\left(h_{1} v^{4}+h_{2} v^{2}+h_{3}\right) \cdot(1-x)+G_{\max } x}
$$

where $h_{1}, h_{2}$ and $h_{3}$ are parameters for fitting the memristor model and $G_{\max }$ is the maximal conductance of the memristor in ON state [17]. The state differential equation of the considered memristor model is [26], [27]:

$$
\frac{d x}{d t}=\left[\begin{array}{l}
\exp \left(\frac{v i}{\sigma_{P}}\right) \exp \left(-\frac{x^{2}}{x_{O N}^{2}}\right) s(v) \\
\left(k_{1} v^{3}+k_{2} v\right)+\sinh \left(\frac{v}{\sigma_{O F F}}\right) A \cdot s(-v) \\
\exp \left(-\frac{x_{\text {OFF }}^{2}}{x^{2}}\right) \exp \left(\frac{1}{1+\beta i v}\right) .
\end{array}\right] f_{\text {win }}
$$

where $A, k_{1}, k_{2}, x_{O N}, x_{O F F}, \sigma_{P}, \beta$, and $\sigma_{O F F}$ are tuning parameters and $f_{\text {win }}$ is a window function for limitation the state variable [16], [26]. The applied smooth and differentiable logistic function $s(v)$ is:

$$
s(v)=0.5\left[1+v\left(v^{2}+m\right)^{-0.5}\right]
$$

where $m$ is a constant with usual value between 50 and 1000. By the change of the transfer coefficient $k_{v}$, a scaling procedure of the synaptic weights in a broad range is obtained. The resistors $R_{2}=R_{3}=300$ $\Omega$ are used for limitation the drain currents of the MOS transistors $T_{1}$ and $T_{2}$ and for determination of their operating points. The resistor $\mathrm{R}_{6}$ with a value of $100 \Omega$ is connected between the source electrodes of the MOS transistors and the ground electrode [26]. This resistor is applied in the circuit for introducing a negative voltage feedback.

\section{RESUlTS AND DisCUSSION}

The risk factors related to the development of COVID-19 disease on a selected group of potential patients has been analyzed, using a memristor neural network with a supervised learning. Unsupervised $\mathrm{k}$-means clustering is also applied for conducting of additional analyses and for comparison the results. A preliminary statistical processing and crossvalidation of the data is made before the training and testing of the neural network for better performance and for deriving more realistic results. A cubic support vector machine (SVM) is applied for the data analyses.

The data containing fifteen significant medical indicators for the potential patients are placed in a table containing data records for 20400 analyzed objects. The first 14280 objects of the table are used for training the neural network, and the respective results are presented in Fig. 3. The derived confusion matrix represents 7161 patients with confirmed COVID-10 disease and 7119 healthy objects. The accuracy of the derived results in this case is near to $100 \%$.

The obtained true positive rate is about $100 \%$. The positive predictive value is also near to $100 \%$. The respective false discovery rate is near to zero. After the learning stage of the neural network, the next 6120 elements are used for their testing. The obtained error in this case is about $3.9 \%$. The next technique applied for separation of the ill and healthy humans is based on a supervised learning of the neural network. The simulation time of the memristor network is about 8534 ms versus 9732 $\mathrm{ms}$ for a traditional neural network. The obtained error in this case is about $3.4 \%$, which is an advantage of the described memristor-based neural network according to the additionally applied machine learning technique.

The described techniques for separation of ill and healthy patients give similar results. Several additional analyses for the distribution of the ill by COVID-19 humans according to their basic properties - age, gender, systolic and diastolic pressure, heart disease, hypertension and cancer are also conducted. The considered analyses represent a large group of patients with COVID illness between 21 and 85 years, having additional cancer symptoms and heart diseases. The COVID-19 illness is mainly observed in patients older than 65 years with related systolic pressure from 75 to 79 . About $47 \%$ of the ill humans are female. About $40 \%$ of the patients with confirmed COVID-19 disease are with diabetes and near to $50 \%$ of them are with cancer disease. 


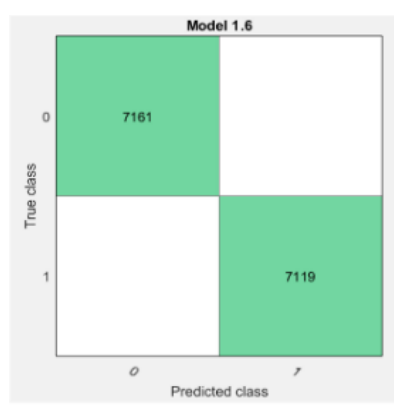

a)

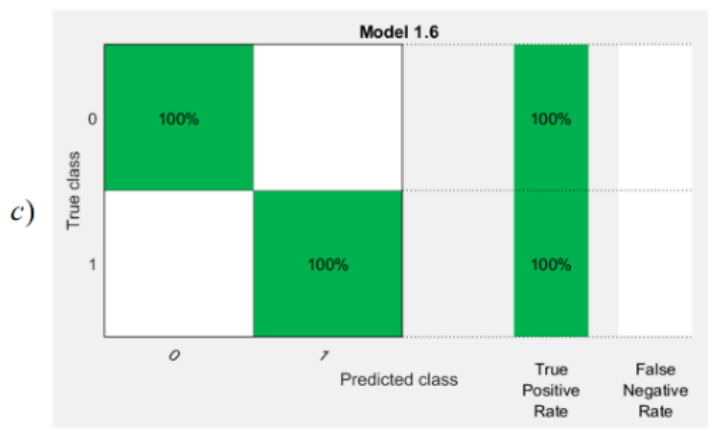

Fig. 3 a) Number of observations; b) Positive predictive values discovery rates; c) True positive rates and false rates

\section{CONCLUSION}

In this work an evaluation of the basic risk factors on a group of potential patients with COVID-19 illness is conducted using a memristorbased neural network. A modified synaptic circuit based on tantalum oxide memristor is applied. The operation of the considered synaptic circuit is founded on a comparison of the currents flowing through the memristor and a resistor, connected in a current divider. A differential amplifier with MOS transistors is also used for additional scaling of the synaptic weights.

An advantage of the proposed synaptic circuit is its ability to ensure positive, zero and negative weights. Another advantage of the offered device is the minimal number of memristors per synapse. For the conducted analyses, an improved and modified model of a tantalum oxide memristor is applied. The considered memristor-based feed forward neural network successfully classifies a set of analyzed people in two categories (ill and healthy potential patients) with a minimal error.

Additional classification of the applied data set is conducted, using a traditional neural network, and a technique for $\mathrm{k}$-means data clustering. The obtained results confirm the proper functionality of the applied feed-forward memristor network for pattern classification and for approximate COVID-19 prediction using several medical indicators for risk factors. The proposed neural network has higher operating speed than its traditional analogue. The potential patients in risk groups are correctly recognized by the considered memristor network using additional medical indicators and the main additional medical features for the development of COVID-19 are evaluated.

\section{ACKNOWLEDGEMENT}

The presented results were obtained under a project funded by the research grant at Technical University of Sofia under project № 202ПД0029-8, "Application of artificial intelligence in recruiting patients for clinical trials" for 2020.

\section{REFERENCES:}

[1] Coronavirus disease (COVID-19) pandemic., https://www.who.int/emergencies/diseases/no vel-coronavirus-2019?gclid=EAIaIQobChM IqJzz54XG7wIVjNSyCh3uEwDREAAYAS AAEgIg5PD_BwE.

[2] Coronavirus disease 2019 (COVID-19)., https://www.mayoclinic.org/diseases-conditio ns/coronavirus/symptomscauses/syc20479963

[3] People with Certain Medical Conditions, https://cdc.gov/coronavirus/2019ncov/needext ra-precautions/people-with-medical-condition s.html.

[4] Castelnuovo, A., Bonaccio, M., Costanzo, S., "Common cardiovascular risk factors and inhospital mortality in 3,894 patients with COVID-19: survival analysis and machine learning-based findings from the multicentre Italian CORIST study", Nutrition, Metabolism and Cardiovascular Diseases, Vol. 30, Issue 11, 30 October 2020, pp. 1899 - 1913.

[5] Anastassopoulou C., Russo L., Tsakris A., Siettos C., "Data-based analysis, modelling and forecasting of the COVID-19 outbreak," PLoS ONE 15 (3), https://doi.org/10.1371/ journal.pone.023 0405, pp. $1-21$.

[6] Alsaeed N., Alqaissi E., Siddiqui M., "An agent-based simulation of the SIRD model of COVID-19 spread," Int. J. Biol. and Biomed. Eng., DOI:10.46300/91011.2020.14.28, Vol. 14, 2020, pp. $210-217$.

[7] Yadav M., Perumal M., Srinivas M., „Analysis on novel coronavirus (COVID-19) using machine learning methods," Elsevier, Chaos, Solitons and Fractals, Nonlinear Science, and Nonequilibrium and Complex Phenomena, Vol. 139, No 110050, pp. 1 - 12. 
[8] Yang L., Liu S., Liu J., Zhang Z., Wan X., Huang B., Chen Y., Zhang Y., "COVID-19: immunopathogenesis and Immunotherapeu tics," Springer Nature Signal Transduction and Targeted Therapy, 128, 2020 https://doi.org/ 10.1038/s41392-020-00243-2, pp. $1-8$.

[9] Bishop, Ch., "Neural Networks for Pattern Recognition," Clarendon Press, Oxford, ISBN 014853864 2, 1995, pp. 1 - 482.

[10] Martin, A., Bartlett P., "Neural network learning: Theoretical foundations," Cambridge University Press, ISBN 978-0521-11862-0, 2009, pp. 404.

[11] Kadaikar A., Pan Y., Zhang Q., CondeCespedes P., Trocan M., Amiel F., Guinot B., "Variable Complexity Neural Networks Comparison for Pollen Classification," Int. J. Biol. Biom. Eng., Vol. 13, 2019, ISSN: 19984510, pp. $38-45$.

[12] Adhikari S. P., Yang C., Kim H., Chua L. O., "Memristor bridge synapse-based neural network and its learning," IEEE Transactions on Neural Networks and Learning Systems, 23 (9) (2012), DOI: 10.1109/TNNLS.2012.22 04770, pp. 1426-1435.

[13] Qinghui Hong, Liang Zhao, Xiaoping Wang., "Novel circuit designs of memristor synapse and neuron," Elsevier, Neurocomputing., 2019, doi: 10.1016/j.neucom.2018.11.043, pp. $11-16$.

[14] Mladenov, V. "Advanced Memristor Modeling - Memristor Circuits and Networks," MDPI Basel, Switzerland, ISBN 978-3-03897-104-7 (Hbk), pp. 1 - 172, doi:10.3390/books978-3-03897-103-0, 2019.

[15] Chua, L., "Memristor - The missing circuit element," IEEE Transactions on Circuit Theory, Vol. 18, Issue: 5, September 1971, , DOI:10.1109/TCT.1971.1083337, pp. 507519.

[16] Strukov, D., Snider, G., Stewart, D., Williams, R. S., "The missing memristor found," Nature Letters., Vol. 453, 2008, pp. $80-83$.

[17] Strachan, J., Torrezan, A., Miao, F., Pickett, M., Yang, J., Yi, W., Medeiros-Ribeiro, G., Williams, R. S., "State Dynamics and Modeling of Tantalum Oxide Memristors," IEEE Transactions on Electron Devices, Vol. 60, No. 7, July 2013, pp. 2194 - 2202.

[18] Xudong, X., Wen, S., Zeng, Z., Huang, T., "Memristor-based circuit implementation of pulse-coupled neural network with dynamical threshold generators," Neurocomputing 284, (2018), pp. 10-16.

[19] Zhang, Y., Wang, X. and Friedman, E.G., "Memristor-based circuit design for multilayer neural networks," IEEE Transactions on Circuits and Systems I: Regular Papers, 65(2), DOI: 10.1109/TCSI.2017.2729787, August 2017, pp.677-686.

[20] Tran, T. "Simulations of Artificial Neural Network with Memristive Devices," A thesis in Electrical Eng., Boise St.Uni., 2012, https://scholarworks.boisestate.edu/cgi/view content.cgi?referer=https://scholar.google.co $\mathrm{m} / \&$ httpsredir $=1 \&$ article $=1337 \&$ context $=\mathrm{td}$.

[21] Campbell, K., Kolton, T. D., Elisa H. S., "Pulse Shape and Timing Dependence on the Spike-Timing Dependent Plasticity Response of Ion-Conducting Memristors as Synapses," Frontiers in Bioengineering and Biotechnology, 2016, Vol. 4, pp. $1-11$.

[22] Mladenov, V., "A Modified Tantalum Oxide Memristor Model for Neural Networks with Memristor-Based Synapses," IEEE Proceeding of IEEE Conference MOCAST, DOI:10.1109/MOCAST49295.2020.9200238, 2020, pp.1-4.

[23] Ascoli, A., R. Tetzlaff, L. Chua, "Robust Simulation of a $\mathrm{TaO}$ Memristor Model," Radioengineering, Volume 24, No. 2, June 2015, pp. $384-392$.

[24] Kang M., Shin S., Jung J., Kim Y., "Stress classification using k-means clustering and heart rate variability from electrocardiogram," Int. J. Bio. Biomed. Eng., Vol. 14, 2020, pp. $251-254$.

[25] Yang, Y., Seung L. C., "Circuit Systems with MATLAB and PSpice," John Wiley \& Sons, 2008, ISBN 978-04-7082-240-1.

[26] Mladenov, V., "A Unified and Open LTSPICE Memristor Model Library," MDPI Electronics, 2021, doi: 10.3390/electronics 10131594, 10(13): 1594, pp. 1 - 27.

[27] Mladenov, V., Kirilov, S., "A Simplified Model of Tantalum Oxide Based Memristor and Application in Memory Crossbars," IEEE Proceeding of MOCAST 2021 Conference, Thessaloniki, Greece, DOI: 10.1109/ MOCAST52088.2021.9493384, pp. 1-4.

[28] Drake K., Lu T., Kamrul M., Majumdar H., and Campbell K., "Comparison of the Electrical Response of $\mathrm{Cu}$ and $\mathrm{Ag}$ IonConducting SDC Memristors Over the Temperature Range $6 \mathrm{~K}$ to $300 \mathrm{~K}$," MDPI 
Micromachines 2019, 10, 663; doi:10.3390/mi10100663, pp. $1-15$.

Valeri Mladenov carried out the mathematical and formal analysis, the design and methodology, the preparation and editing of the manuscript.

Ognyan Nakov organized the investigations, the preparation, and the statistical analysis in the present work.

Violeta Todorova and Stoyan Kirilov made the implementation of the algorithms, the analysis, and the technical preparation of the paper.

\section{Creative Commons Attribution}

\section{License 4.0 (Attribution 4.0}

International , CC BY 4.0)

This article is published under the terms of the Creative Commons Attribution License $4.0 \mathrm{https}: / /$ creativecommons.org/licenses/by/4.0/deed.en_US 